American Journal of Applied Sciences 7 (4): 500-508, 2010

ISSN 1546-9239

(C) 2010Science Publications

\title{
Gamma Irradiation Synthesis and Influence the Optical and Thermal Properties of Cadmium Sulfide (CdS)/Poly (Vinyl Pyrolidone) Nanocomposites
}

\author{
${ }^{1}$ Shawkat Salameh Gasaymeh, ${ }^{1}$ Shahidan Radiman, ${ }^{2}$ Lee Yook Heng and ${ }^{3}$ Elias Saion \\ ${ }^{1}$ Department of Nuclear Science, Faculty of Science and Technology, School of Applied Physics, \\ University of Kebangsaan Malaysia, 43600 (UKM) Bangi Selangor, Malaysia \\ ${ }^{2}$ Faculty of Science and Technology, School of Chemistry and Food Science, \\ UKM-43600 Bangi Selangor, Malaysia \\ ${ }^{3}$ Department of Applied Physics, Faculty of Science, Putra University, \\ UPM-43400 Serdang, Selangor Malaysia
}

\begin{abstract}
Problem statement: Gamma irradiation has been successfully used to prepare a spherical cadmium sulfide CdS/Poly Vinylpyrolidone (PVP) quantum dots nanoparticles with enhancement of their optical band gap energy and thermal properties at room temperature and under ambient pressure. Sodium thiosulfate was used as a sulfur source in an aqueous solution. The formation of lower band gap energy of CdS/PVP nanoparticles and thermal stability can be controlled by using different irradiation doses. TEM images showed that the CdS/PVP particle size tends to be smaller and with better distribution as irradiation dose increases. Approach: Gamma $(\gamma)$ irradiation offered many advantages for the preparation of metal nanoparticles by producing large number of hydrated electrons during $\gamma$-ray irradiation, which can reduce the metal ions to zero valiant metal particles. Results: CdS/PVP nanoparticles were successfully prepared in one-step by $\gamma$-irradiation technique in an aqueous system at room temperature and under ambient pressure. The particle size was found to be less than $10 \mathrm{~nm}$ based on the Transmission Electron Microscopy (TEM) that depended on the irradiation doses value, which showed a well distribution with a controlled size as doses change. The presence of PVP polymer was considered an important reason that influenced the shape and the distribution of those nanoparticles. The optical band gap energy of those nanoparticles was calculated by using the UV-VIS absorption spectra. Thermal analysis TGA showed that the composite had a higher degradation temperature than the PVP alone. A possible mechanism of the formation of cadmium sulfide by irradiation system was proposed. Conclusion: This result indicated that $\mathrm{CdSO}_{4}$ can effectively dope PVP and enhance the optical and thermal properties. In addition, $\gamma$-irradiation is an effective technique for preparing inorganic/organic nanocomposites.
\end{abstract}

Key words: CdS/PVP, band gap energy, TEM, thermal properties, gamma irradiation

\section{INTRODUCTION}

Nanotechnologies are now poised to revolutionize the electronic, chemical and biotechnology industries and biomedical fields. There are many interesting areas in nanotechnology. One of the most important aspects of this field is the preparation and development of nanomaterials, such as nanoparticles. There has been a variety of techniques for preparing different types of nanoparticles. The formalism confirming the scaling laws of materials with size tunable properties is couched in the physics language of "Quantum Size Effects," (QSE), or semiconductor crystallites that are small in comparison to bulck electron delocalization lengths $(10-100 \AA)$ are the subject of extensive investigation (Murray et al., 1993). The Schrödinger wave equation is solved for an electron and hole in a box having either 1D, 2D or 3D and spatial dimensions of the order of the Bohr radius of the electron, hole or exciton (electron-hole pair) (VanZeghbroeck, 2007; Hu et al., 1990; Chestnoy et al., 1986; Murray et al., 1993). In this intermediate size range between molecular and bulk matter, called the nanoscale, individual energy states of molecules and continuous energy bands of solids become discrete and their energy separations display an analytic dependence on the spatial dimension

Corresponding Author: Shawkat Salameh Gasaymeh, Department of Nuclear Science, Faculty of Science and Technology, School of Applied Physics, University of Kebangsaan Malaysia, 43600 (UKM) Bangi Selangor, Malaysia Tel: +60173928906/ 
of the material. Quantum confinement, the widening HOMO LUMO gap with decreasing crystallite size and its implications for the electronic structure and photophysics of the crystallites have generated considerable interest (Murray et al., 1993). The synthesis and characterization of semiconductors have attracted significant interest and still are the subject of intense investigation owing to their important nonlinear properties, luminescent properties, quantum size effects and other important physical and chemical properties (Goldstein et al., 1992). Cadmium sulfide is one the most interesting semiconductors due to its high photosensitivity and attractive application in photoconducting cells. Gamma-irradiation has been successfully used to prepare metal/polymer nanocomposites with a one-step synthesis using $\gamma$ irradiation in aqueous solutions (Karim et al., 2007; Qiao et al., 1999; Zhengping et al., 2000; Jan et al., 2004), with detail of characterization.

A possible mechanism of the formation of $\mathrm{CdS}$ quantum dots nanoparticles by $\gamma$-irradiation system is proposed in Scheme 1, for more detail refer to Qiao et al. (1999). The color of the irradiated samples were changed from colorless before irradiation to yellow or golden yellow to darker in color as the dose increases. This result may be due to the reduction of $\mathrm{CdSO}_{4}$ to cadmium nanoparticles in PVP solution that acts as a binder or protective agent, which restricts the mobility of the $\mathrm{Cd}$ ions during chemical reaction, prevents aggregation among the CdS quantum dots nanoparticles and limits the size of CdS nanoparticles (Bogle et al., 2006; Khanna et al., 2005). In recent years, studies on the electrical and optical properties of polymers have attracted much attention in view of their application in optical devices. The optical properties of polymers can be suitably modified by the addition of dopants depending on their reactivity with the host matrix. Although handful researches has been reported on the charge carrier transport and optical properties of doped polymers, but to the best of our knowledge no work is available on doped PVP. PVP is a potentially useful material in which its dielectric strength is very high; furthermore, it has a good charge storage capacity and dopant-dependent optical properties. The incorporation of inorganic/organic is believed to remarkably improve a wide range of properties of the polymers due to the nanosized dispersion. Among these properties, the enhancement of thermal property is one that has been observed in many polymer nanocomposites. It was reported that incorporation of inorganic/organic significantly elevates the glass transition temperature and the degradation temperature of the neat polymer (Park et al., 2002; Lebaron et al., 1999).

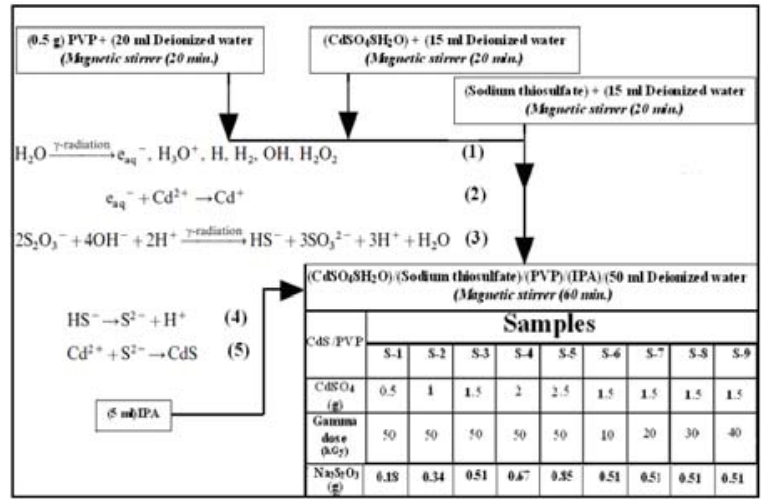

Scheme 1: The procedures and the quantity of the prepared CdS quantum dots/PVP nanocomposites with a proposed formation of CdS quantum dots

In this study, we report the thermal stability and the optical band gap depends on the value of dose and/or the concentration of $\mathrm{CdSO}_{4}$ in the system. We found a decrease in the optical band gap energy as dose goes higher and thermal stable the composite become. Moreover the size and particle distribution were controlled by irradiation dose.

The band gap of a material is defined as the energy distance between the valence and Conduction Bands (CB). The smaller the band gap, the more electrically conductive a material will be. Metals have small band gaps whereas insulators have larger band gaps that explain why there are so few electrons found in their conduction bands. There is not sufficient thermal energy to excite electrons from the valence band to the conduction band in the case of insulators. Semiconductors occupy a middle ground and have tractable band gaps, which when given enough thermal energy can excite electrons into the conduction band. One can also add dopants to semiconductors like cadmium sulfide quantum dots which make a semiconductor behave more like a conductor by introducing additional energy levels within the band gap. The key difference to note is the difference between discrete atomic or molecular orbitals and the bands in a solid. The organization of energy levels is quite different between nanomaterials and bulk materials in those nanomaterials have larger band gaps and consist of more discrete energy levels. This observation may be attributed to the various quantum confinement effects within nanoparticles whose sizes can be as small as only a few dozen atoms wide (couple of nanometers). Generally, the optical band gap energy in a semiconductors is determined by plotting 
absorption coefficient $\alpha(\mathrm{v})$ as $(\alpha(\mathrm{v}) \mathrm{hv})^{1 / \mathrm{m}} \mathrm{Vs}$ hv where $\mathrm{m}$ represents the nature of the transition and $\mathrm{hv}$ is the photon energy. In addition, $\mathrm{m}$ may have different values, such as $1 / 2,2,3 / 2$ or 3 for allowed direct, allowed indirect, forbidden direct and forbidden indirect transitions respectively.

\section{MATERIALS AND METHODS}

Silver nitrate $\left(\mathrm{CdSO}_{4} 8 \mathrm{H}_{2} \mathrm{O}\right)$, Sodium thiosulfate $\mathrm{Na}_{2} \mathrm{~S}_{2} \mathrm{O}_{3} 5 \mathrm{H}_{2} \mathrm{O}$, Polyvinilpirrolidone PVP $\mathrm{Mw}=10.000$ (Aldrich) and other chemicals were used as received. Deionized water was prepared in the laboratory, Isopropyl Alcohol (IPA) was used as radical scavenger in all samples.

CdS/PVP nanocomposites have been synthesized by using $\gamma$-irradiation process. For this purpose, procedures were done in a clean room by dissolving different ratios of $\left(\mathrm{CdSO}_{4}\right)$ as illustrated in Scheme 1, in deionized water and maintained under vigorous stirring for $20 \mathrm{~min}$. until a homogeneous solution was obtained. At the same time, PVP was dissolved in $\mathrm{H}_{2} \mathrm{O}$ and kept under magnetic stirrer for 20 min. The $\mathrm{Na}_{2} \mathrm{~S}_{2} \mathrm{O}_{3}$ was dissolved separately also by $\mathrm{H}_{2} \mathrm{O}$ under magnetic stirrer and then the three different solutions were added together and IPA was then added to the mixture and stirred for thirty minutes more. Finally, the homogenous mixture was bubbled with nitrogen before sending it to $\gamma$-cell to be irradiated at different doses. The amount of PVP was $0.5 \mathrm{~g}$ for all samples and the molar ratio $\left(\mathrm{CdSO}_{4}\right) / \mathrm{Na}_{2} \mathrm{~S}_{2} \mathrm{O}_{3}$ was constant in all samples.

The purified yellow or/and the dark darker yellow precipitate was centrifuged and washed in deionized water and ethanol several times and dried in oven at $70^{\circ} \mathrm{C}$ for 7 hours to be ready for further characterizations. It is important to mention the color of the CdS/PVP nanocomposites solution is yellow and goes to darker as dose increases or/and as the concentration of $\mathrm{CdSO}_{4}$ increase which indicates to more reduction of $\mathrm{CdSO}_{4}$ to $\mathrm{CdS}$ nanoparticles.

\section{RESULTS}

Effect of $\gamma$-irradiation and $\mathrm{CdSO}_{4}$ concentration on the Morphology by TEM: In order to study the effect of irradiation dose on the reduction of $\mathrm{CdSO}_{4}$ to $\mathrm{CdS}$ nanoparticles in PVP matrix TEM images at different doses was taken for CdS/PVP nanocomposites.

Figure 1 and 2 show the TEM micrograph of CdS/PVP nanocomposites prepared at 50 and $40 \mathrm{kGy}$ respectively. The result revealed a spherical nanoparticle of CdS in both doses and smaller size was obtained as dose increased. Moreover, the result revealed that the higher $\gamma$-doses lead to more reduction of $\mathrm{CdSO}_{4}$ to $\mathrm{CdS}$ nanoparticles in PVP matrix.

The function of PVP in the CdS/PVP composites is not only as a binder but it prevents the process of agglomeration of CdS nanoparticles (Bogle et al., 2006; Kang et al., 2006) and limits the diameter of the nanoparticles that formed. The polymer was used in this study due to the hydrophilic nature of PVP that protects the surface of the CdS nanoparticles. The average particle size CdS/PVP at different dose with a fixed amount of $1.5 \mathrm{~g}$ of $\mathrm{CdSO}_{4}$ in our experiment was estimated to be ranging from around $8 \mathrm{~nm}$ at $50 \mathrm{kGy}$ with well and large distribution to around $100 \mathrm{~nm}$ at 40 kGy as shown in Fig. 1 and 2 respectively.

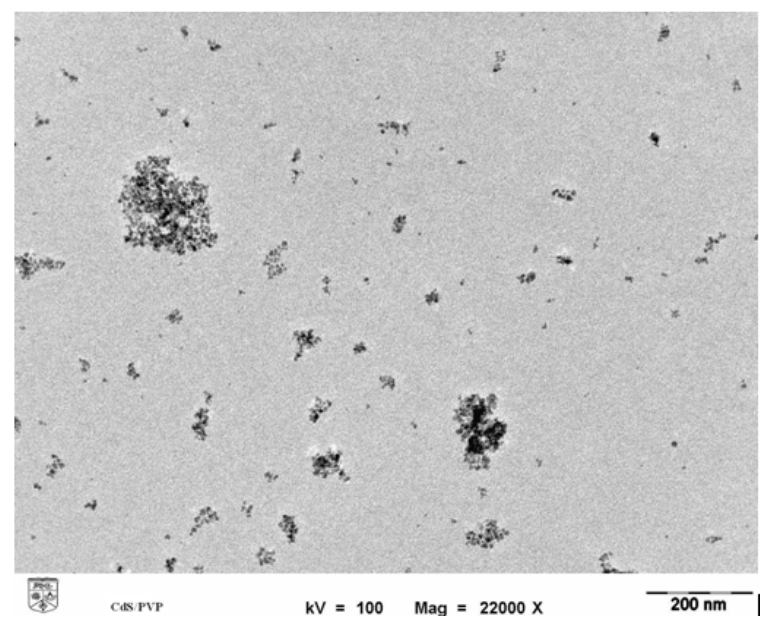

Fig. 1: TEM micrograph of CdS nanoparticles in PVP matrix at $50 \mathrm{kGy}$ with $1.5 \mathrm{~g}$ of $\mathrm{CdSO}_{4}$

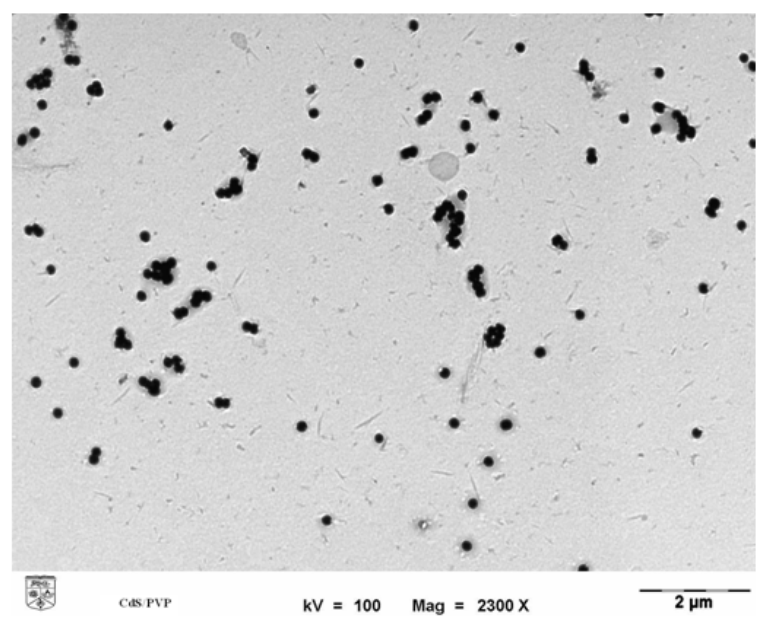

Fig. 2: TEM micrograph of CdS nanoparticles in PVP matrix at $40 \mathrm{kGy}$ with $1.5 \mathrm{~g}$ of $\mathrm{CdSO}_{4}$ 
This indicates that the diameters of nanomaterials can be controlled directly by the appropriate selection of irradiation dose. In addition, nanomaterials obtained by our method all show similar range of diameters up to several nanometers, moreover different shape can be produced by $\gamma$-irradiation Ag/polystyrene nanorod was prepared by our research group (Jan et al., 2004) by using surfactants. Further, the formation, size and distribution of CdS nanoparticles were controlled by varying the dose as shown in the TEM images, which satisfies our objectives.

UV-visible spectophotometry is one of basic techniques to study the quantum confinement effects. The absorption spectrum is basis for sizing and gives an approximate estimate of the size distribution from the sharpness of the absorption features. The absorption bands shift to higher energies with decreasing quantum dot sizes, "blue shift". Generally, the optical band gap is blue shifting dramatically from the bulk size amount to the quantum sizes. In addition, bellow exciton Bohr radius the absorption spectra shows a fine structure. Appearance of a fine absorption spectrum is due to the presence of discrete energy levels. Since the exciton levels become delocalized over the whole quantum dot the absorption spectra of this quantum dots will be affect by the exciton transitions (VanZeghbroeck, 2007; Hu et al., 1990; Chestnoy et al., 1986; Murray et al., 1993). UV-vis characterizes the optical properties of $\mathrm{CdS}$ quantum dots shown in Fig. 3A for $1.5 \mathrm{~g}$ of $\mathrm{CdSO}_{4}$ in PVP matrix prepared at different dose ranging from 10 to $50 \mathrm{kGy}$ in step of $10 \mathrm{kGy}$. Figure $3 \mathrm{~B}$ shows the UV-vis characterizes the optical properties of CdS/PVP nanocomposites for 0.5-2.5 $\mathrm{g}$ in step of $0.5 \mathrm{~g}$ of $\mathrm{CdSO}_{4}$ at $447 \mathrm{~nm}$ in PVP solution at dose of $50 \mathrm{kGy}$. All samples were dispersed in the absolute ethanol and UV-vis measurement carried out by using the absolute ethanol as reference. The obvious absorption peak around 440-470 $\mathrm{nm}$ can be assigned to the first excitation of $\mathrm{CdS}$ and compared to that of $\mathrm{CdS}$ nanoparticles $(\approx 470 \mathrm{~nm})$. As shown in Fig. 3A the band edge $(\approx 440 \mathrm{~nm})$ of the first sample (3-wt $\%$ of $\left.\mathrm{CdSO}_{4}\right)$, a higher absorption and wave length for the other samples as we increase the amount of $\mathrm{CdSO}_{4}$, (from 6$16 \mathrm{wt} \%$ of $\mathrm{CdSO}_{4}$ ). This shift with higher intensity as the $\mathrm{CdSO}_{4}$ concentration increase, indicate that $\mathrm{CdS}$ nanoparticles in the dispersion nucleate to form larger size nanocrystallites.

On the other hand, as shown in the same Fig. 3B, there is a blue shifted as $\gamma$-dose increase, indicating that $\mathrm{CdS}$ nanoparticles are mono-dispersed with smaller size as dose increases, but in the other case as $\mathrm{CdSO}_{4}$ increase a red shift occurs shown in $3 \mathrm{~A}$ indicating the size go larger as $\mathrm{CdSO}_{4}$ increases. It is obvious to make a conclusion that the stable dispersion of CdS has a quantum effect. Comparing our result to the absorption peak of bulk CdS crystal at $513 \mathrm{~nm}$, (band gap $2.4 \mathrm{eV}$ ), the blue shift of absorption peak can be attributed to the quantum size effect.

Figure $4 \mathrm{~A}$ and $\mathrm{B}$, shows the a plot of absorbance vs. $\mathrm{CdSO}_{4}$ concentration, the result an increase in the intensity from 0.4 for $0.5 \mathrm{~g}$ to 2.2 for the $2.5 \mathrm{~g}$, indicating more reduction of $\mathrm{CdSO}_{4}$ to $\mathrm{CdS}$ nanoparticles. In the other case of increasing the $\gamma$-dose from 10-50 kGy the absorbance increase from 0.6-2.3 indicating same result as $\gamma$-dose, increase reduction of $\mathrm{CdSO}_{4}$ to $\mathrm{CdS}$ increase in the PVP solution.

Effect of $\gamma$-irradiation and $\mathrm{CdSO}_{4}$ concentration on the optical band gap energy by using UV-Vis spectra: Fig. 5A shows the direct allowed transition properties using Mott and Davis concept for $1.5 \mathrm{~g}$ of $\mathrm{CdSO}_{4}$ at $447 \mathrm{~nm}$ in PVP solution prepared at different doses ranging from 10-50 kGy in step of $10 \mathrm{kGy}$.

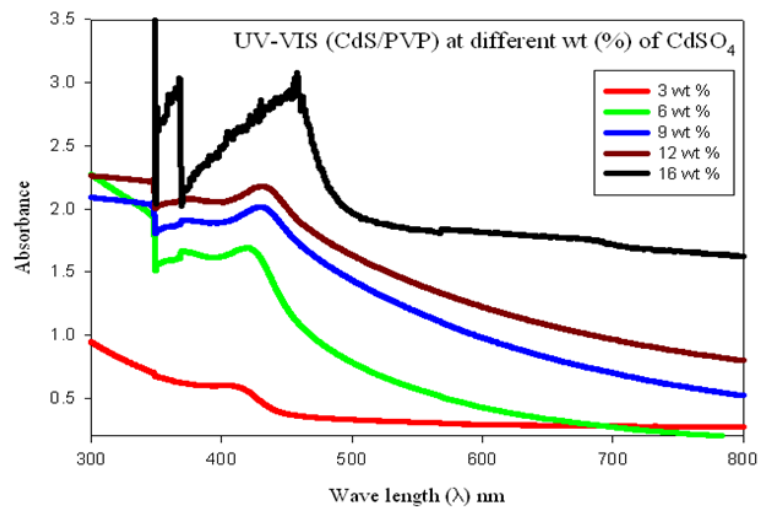

(A)

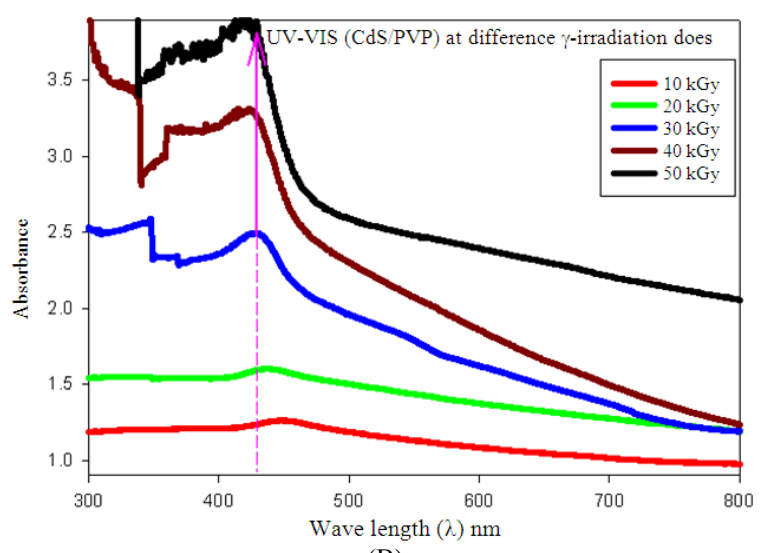

(B)

Fig. 3: UV-vis absorption spectra for different concentration of $\mathrm{CdSO}_{4}$ (A) and for different $\gamma$ doses (B) 


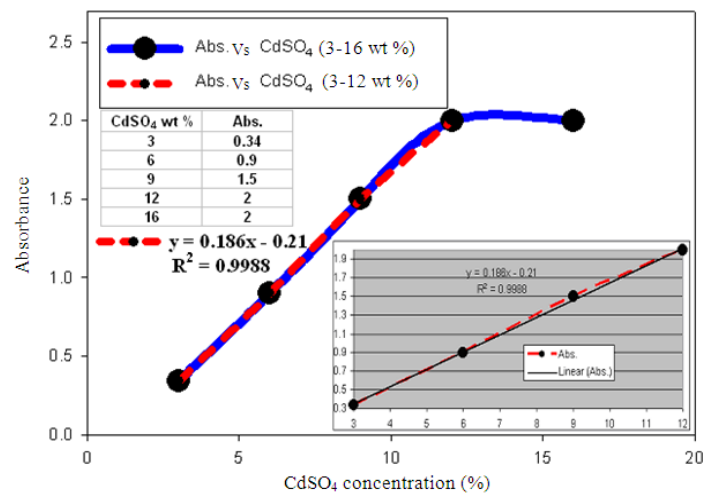

(A)

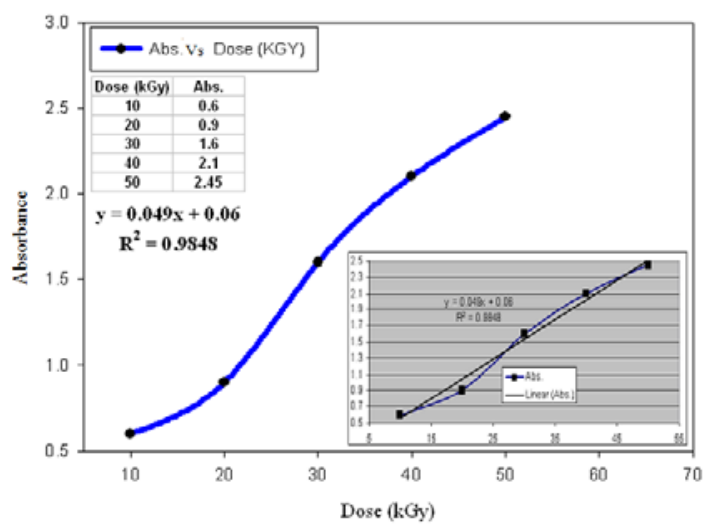

(B)

Fig. 4: Plot of Absorbance Vs the concentration of $\mathrm{CdSO}_{4} \mathrm{wt} \%$ (A) and $\mathrm{Vs}$ the dose-kGy (B)

Figure 5B shows the direct allowed transition properties using same concept for $0.5-2.5 \mathrm{~g}$ in step of $0.5 \mathrm{~g}$ of $\mathrm{CdSO}_{4}$ at $447 \mathrm{~nm}$ in PVP solution prepared at dose of $50 \mathrm{kGy}$. The band gap energy may be determined from the extrapolation of the linear section of the curves to $\mathrm{x}$ axis in which $(\alpha(v) h v)^{2}=0$. This will give the direct band gap energies of absorption of the electrons in the conduction bands of $\mathrm{CdS}$ nanoparticles induced by $\gamma$ irradiation illustrated in Fig. 6A at various doses and Fig. 6B at different $\mathrm{CdSO}_{4}$ amount.

The band gap energy decreases following the radiation dose increment from $2.69 \mathrm{eV}$ at $10 \mathrm{kGy}$ to 2.4 $\mathrm{eV}$ at $50 \mathrm{kGy}$. This decrease in the band gap is attributed to more reduction of $\mathrm{CdSO}_{4}$ to $\mathrm{CdS}$ nanoparticles with smaller size as dose increase (Niu et al., 2003; Devendrappa et al., 2006).

Moreover, the result showed a strong dependence on the concentration of the $\mathrm{CdSO}_{4}$ concentration showing a maximum value for the sample with a concentration of $12 \mathrm{wt} \%$ of the $\mathrm{CdSO}_{4} \mathrm{wt} \%$. Above this concentration (16 wt $\%$ ), the band gap energy increase continuously with increasing $\mathrm{CdSO}_{4}$ concentration.

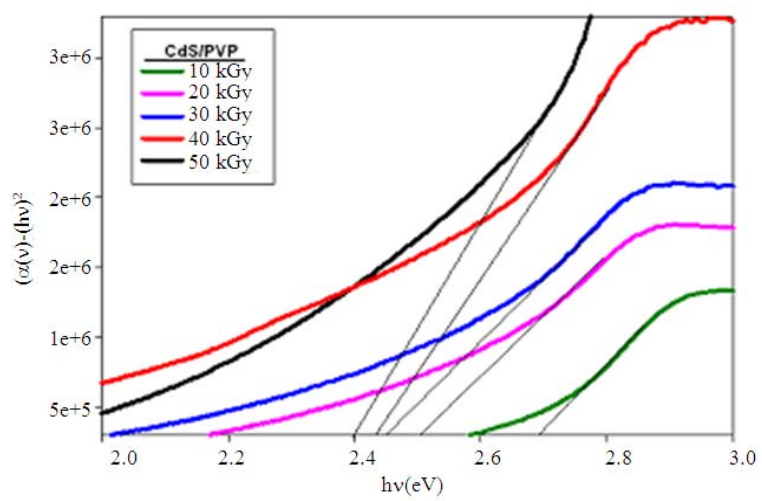

(A)

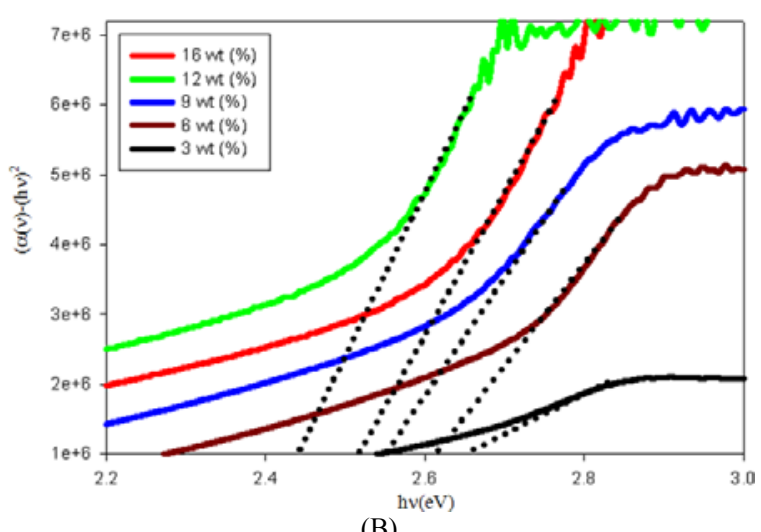

(B)

Fig. 5: (A): Variation of direct allowed transition using Mott and Davis concept for $\mathrm{CdS}$ quantum dots/PVP nanocomposites at $447 \mathrm{~nm}$ at different doses and (B): The variation of band gap energy for CdS quantum dots in PVP at different $\mathrm{CdSO}_{4} \mathrm{wt}^{\%}$

The band gap energy decreases from $2.67 \mathrm{eV}$ at $3 \mathrm{wt} \%$ of $\mathrm{CdSO}_{4}$ to $2.44 \mathrm{eV}$ at $12 \mathrm{wt} \%$ of $\mathrm{CdSO}_{4}$. Further increase of $\mathrm{CdSO}_{4}$ at $16-\mathrm{wt} \%$ the band gap energy showed an increase to be $2.52 \mathrm{eV}$. At higher doses and concentration from 3-12 wt $\%$ there is a decrease in the band gap energy due to the fact that the absorption band shifted slightly to the lower wavelengths. This result showed a significant change in the particle diameters, which were confirmed by the TEM results.

\section{Effect of $\gamma$-irradiation and CdS nanoparticles on the} thermal properties by using TGA: TGA measurements were carried out on the CdS/PVP and pure PVP. A known weight of the samples was heated at a rate of $10^{\circ} \mathrm{C} \mathrm{min} \mathrm{m}^{-1}$ from room temperature up to $600^{\circ} \mathrm{C}$, which is in between the boiling point of the solvent and the degradation temperature of the polymer. 
Am. J. Applied Sci., 7 (4): 500-508, 2010

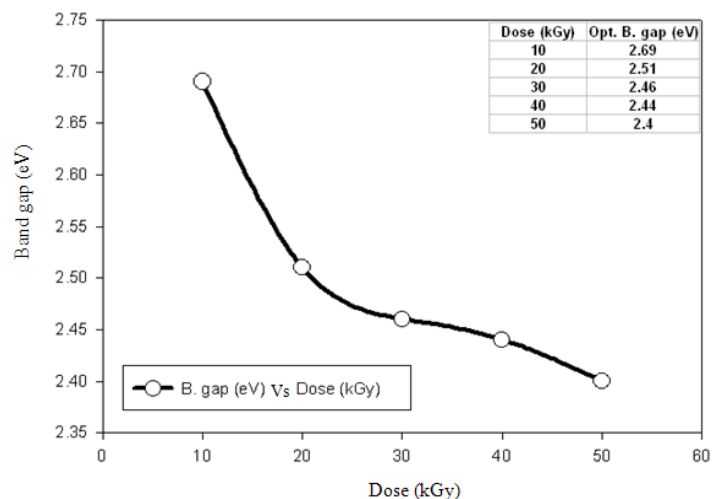

(A)

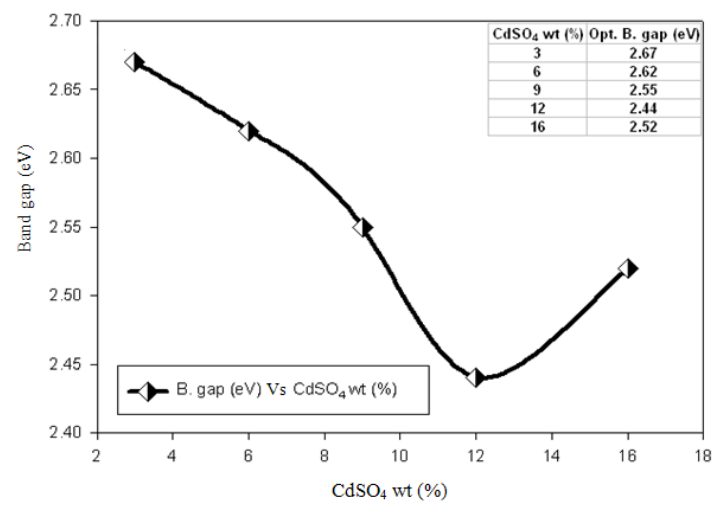

(B)

Fig. 6: (A): Variation of band gap energy for CdS quantum dots in PVP at different doses and (B): The variation of band gap energy for $\mathrm{CdS}$ quantum in PVP at different $\mathrm{CdSO}_{4}$ concentration

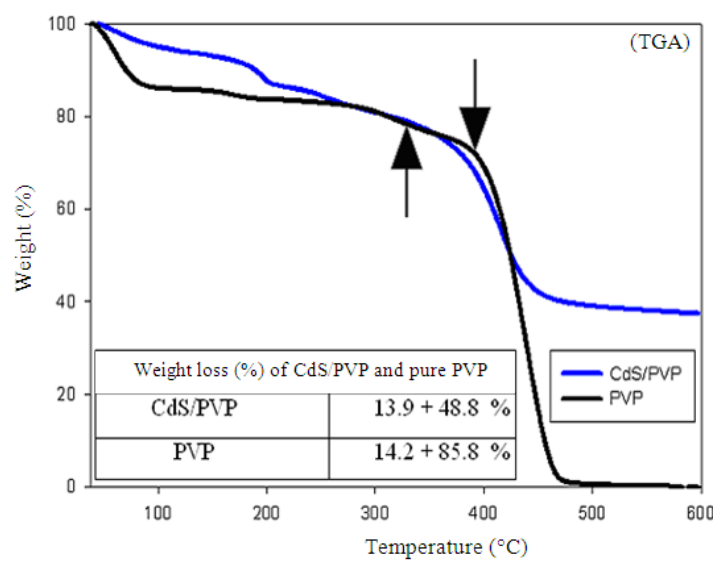

Fig. 7: The TGA thermogram of (a): CdS/PVP nanoparticles and (b): Pure PVP. Where, the weight loss tabulated from TGA thermogram results at first and second stages
Figure 7, shows two distinct stages of weight loss, an initial weight loss was calculated to be around 13.9 and $14.2 \%$ for CdS/PVP and pure PVP respectively in the range of room temperature up to $250^{\circ} \mathrm{C}$.

The weight loss up to this range $\left(250^{\circ} \mathrm{C}\right)$ of temperature is attributed to low molecular weight oligomers, loss of moisture and residual solvent. The second weight loss indicated that the pure PVP began to degrade above $350^{\circ} \mathrm{C}$ and is completely decomposed at above $500^{\circ} \mathrm{C}$. The second major weight loss was attributed to structural decomposition of polymer. The weight loss of CdS/PVP nanoparticles and pure PVP is tabulated in Fig. 7. However, the thermogravimetric analysis of the CdS/PVP nanocomposite showed decomposition profile starting at about $350^{\circ} \mathrm{C}$ and continuing until about $440^{\circ} \mathrm{C}$. This shows that the thermal stability of the polymer is improved due to presence of CdS as nano-filler. This observation is consistent with the results obtained by Mbhele et al. (2003), where it has been shown that the polymer alone starts decomposing at about $280^{\circ} \mathrm{C}$ and its composite with about $<1 \% \mathrm{Ag}$ starts decomposing at higher temperature than the polymer alone, typically about $40^{\circ} \mathrm{C}$ more. In our experiments, though the content of $\mathrm{CdS}$ is expected to be more but it appears that the degradation temperature of our composite is higher to that it is reported.

\section{DISCUSSION}

However, comparing our results with those previous studies may be used as a discussion on the subject. Handful of published researches (Jing et al., 2006; Wu et al., 2005; Jia et al., 2006; Liu et al., 2006) successfully synthesized different nanomaterials using electron irradiation techniques, obtained that the particle size was about $40 \mathrm{~nm}$ and by using $\gamma$-irradiation $30 \mathrm{~nm}$ was achieved for the same sample. Moreover, it was reported in the same experiment that the diameter of particles tends to reduce in size with the increase of dose. Moreover, they were obtained similar result under light irradiation with average size of $100 \mathrm{~nm}$. All the above results indicate that the irradiation is an effective technique for preparation of nanoparticles. It is clear from the band gap energy calculations that the band energy showed a decrease as dose increase. Such decrease in the band gap is attributed to more reduction of $\mathrm{CdSO}_{4}$ to $\mathrm{CdS}$ nanoparticles similar results were observed by (Niu et al., 2003; Devendrappa et al., 2006) and the particle go smaller as dose increase as show by the TEM micrographs. The smaller the size the more clouded of electrons is surrounded. Moreover, as dose or/and $\mathrm{CdSO}_{4}$ concentration increase the more 
reduction of CdS occurs. All these observations are responsible for decreasing the band gap energy.

Furthermore, the intensity of the absorption bands, which is proportional to the number of $\mathrm{CdS}$ nanoparticles, goes higher, indicates that more $\mathrm{CdS}$ nanoparticale are formed as dose increase similar result observed by (Bogle et al., 2006) for different composite of Ag/PVA prepared by electron irradiation and by (Porel et al., 2005) prepared by $\gamma$-rays. The observation of decreasing in band gap for doped samples of CdS/PVP may be attributed to the fact that their charge transfer complexes increase the electrical conductivity by providing additional charges in the polymer lattice due to the corporation of CdS nanoparticles with PVP by the influence of $\gamma$-irradiation.

However, in further addition of $\mathrm{CdSO}_{4}$ concentration above $12 \mathrm{wt} \%$, it leads to segregation of the dopant of $\mathrm{CdS}$ in the host polymer. These molecular aggregates impede the motion of charge carriers resulting in a decrease in their conductivity and hence increased optical band gap energy. Similar results have reported by (Bhat and Kelkar, 1990) on the effect in copper chloride-doping nylon-6 films. These results point to the fact that a dopant concentration up to 12 $\mathrm{wt} \%$ is the critical concentration that gives optimum values for the optical properties. The incorporation of different nanoparticles could effectively improve the electrical, optical and dielectric properties of the polymer composites (Yakuphanoglu et al., 2006; Sarma et al., 2002). These properties are very much sensitive to small changes in the nanoparticles content and in the size and shape of the nanoparticles. It was reported that the nanoparticles themselves could act as conductive junctions between the polymer chains that resulted in an increase of the electrical conductance of the composites (Gangopadhyay and De, 2000; Castillo-Castro et al., 2007) in which leads to a decrease in their optical band gap energy. The morphology and the particle size may play an important role in the optical absorption spectra of the CdS/PVP nanoparticles. The entire above calculated band gap samples have different particle size as shown by the TEM micrographs and the UV absorption spectra. Moreover, irradiation may change the direction of polarization, this change in the direction of polarization lowers the value of dielectric and as a result, their band gap energy will be reduced. In addition, the size was change following the dose increment, the size dependence of the peak position is explained by a sizedependent effective dielectric constant of a metal particle due to the interactions of conduction electrons (Bogle et al., 2006; Tjong and Liang, 2005). When the particles become much smaller than the mean free path of conduction electrons in the bulk metal, the effective mean free path becomes the dimensions of the particle because electrons are scattered at particle surface. Since the mean size of the CdS particles $(\sim 8 \mathrm{~nm})$ produced by our method are much smaller than the mean free path of bulk CdS $(\sim 55 \mathrm{~nm})$, such effect must be significant. The present technique (gamma irradiation) leads to a simultaneous increase of the volume fraction with decreasing size of the CdS nanoparticles as evidence from the TEM micrographs. Irradiation of CdS/PVP samples leads to chemical change in polymer chain, namely, chain scission and cross-linking, which all lead to improve optical properties (Virk and Srivastava, 2001; Steckenreitera et al., 1997; Singh et al., 2008). The inter-particle distance decreased as more $\mathrm{CdS}$ incorporated and as dose increase, both factors can decrease the band gap of the composites. Since the changes in the volume fraction of the particles in the sample leads to a change of the dielectric constant of the composite due to electromagnetic interaction between neighboring particles. This effect is also thought to be responsible for the change of the band gap as has been shown previously by (Bogle et al., 2006; Khanna et al., 2005). The thermal stability is one of the most important and inter related variable, in which must be carefully considered when a material is chosen for the synthesis of organic electronic devices. There appears to be an optimal concentration of $\mathrm{CdS}$ nanoparticles in PVP, which maximizes the thermal properties of the materials. Sample with $1.5 \mathrm{~g}$ of $\mathrm{CdSO}_{4}$ was found to be suitable and thermal stable. Therefore, the optimized CdS/Polymer nanocomposites materials have the potential to produce organic electronic device capable of higher thermal stability than pure polymer, leading to many useful application in organic electronics and nanotechnology.

\section{CONCLUSION}

This result indicates that $\mathrm{CdS}$ nanoparticles can effectively dope PVP and enhance the optical and thermal properties. In addition, $\gamma$-irradiation is an effective technique for preparing inorganic/organic nanocomposites. The improvement in the optical properties may attribute to irradiation with $\gamma$-ray that leads to the formation of $\pi$-electron clouds, with the polarization aligned in the direction of the molecular chains (Bhat and Kelkar, 1990).

\section{ACKNOWLEDGEMENT}

We thank the Faculty of Science and Technology University Kebangsaan Malaysia (UKM) for their 
valuable advice and support. We also acknowledge (UKM) in providing instrumentation facilities and grant UKM-OUP-NBT-28-138/2009, which made it possible for this study to be conducted. In addition, S.S. Gasaymeh would like to thank En. John S. Kasem for his financial support.

\section{REFERENCES}

Bhat, N.V. and D.S. Kelkar, 1990. Investigation of electrical conductivity of nylon-6 films doped with copper chloride. J. Phys. D. Applied Phys. 23: 899-902. DOI: $10.1088 / 0022-3727 / 23 / 7 / 022$

Bogle, K.A., S.D. Dhole and V.N. Bhoraskar, 2006. Silver nanoparticles: Synthesis and size control by electron irradiation. Nanotechnology, 17: 3204-3208. DOI: 10.1088/0957-4484/17/13/021

Castillo-Castro, T.D., E. Larios-Rodriguez, Z. MolinaArenas, M.M. Castillo-Ortega and J. Tanori, 2007. Synthesis and characterization of metallic nanoparticles and their incorporation into electroconductive polymer composites. Composites: Part A. Applied Sci. Manuf., 38: 107-113. DOI: 10.1016/j.compositesa.2006.01.011

Chestnoy, N., T.D. Harris, R. Hull and L.E. Brus, 1986. Luminescence and photophysics of cadmium sulfide semiconductor clusters: The nature of the emitting electronic state. J. Phys. Chem., 90: 3393-3399. DOI: $10.1021 / \mathrm{j} 100406 \mathrm{a} 018$

Devendrappa, H., U.V. Subba Rao and M.V.N. Ambika Prasad, 2006. Study of dc conductivity and battery application of polyethylene oxide/polyaniline and its composites. J. Power Sour., 155: 368-374. DOI: 10.1016/j.jpowsour.2005.05.014

Gangopadhyay, R. and A. De, 2000. Conducting polymer nanocomposites: A brief overview. Chem. Mater. 12: 608-622. http://pubs.acs.org/doi/abs/10.1021/cm990537f

Goldstein, A.N., C.M. Echer and A.P. Alivisatos, 1992. Melting in semiconductor nanocrystals. Sci., Ser., 256: 1425-427. http://www.jstor.org/stable/2876942

Hu, Y.Z., M. Lindberg and S.W. Koch, 1990. Theory of optically excited intrinsic semiconductor quantum dots. Phys. Rev., 42: 1713-1723. DOI: 10.1103/PhysRevB.42.1713

Jan, L.S., S. Radiman, M.A. Siddig, S.V. Muniandy and M.A. Hamid et al., 2004. Preparation of nanoparticles of polystyrene and polyaniline by $\gamma$ irradiation in lyotropic liquid crystal. Coll. Surfaces Physicol. Eng. Aspects, 251: 43-52. DOI: 10.1016/j.colsurfa.2004.09.025
Jia, H., J. Zeng, W. Song, J, An and B. Zhao, 2006. Preparation of silver nanoparticles by photoreduction for surface-enhanced Raman scattering. Thin Solid Films, 496: 281-287. DOI: 10.1016/j.tsf.2005.08.359

Jing, L., L. Zhu, Y. Wu, Y. Harima and A. Zhang et al., 2006. Hybrid composites of conductive polyaniline and nanocrystalline titanium oxide prepared via self-assembling and graft polymerization. Polymer, 47: 7361-7367. DOI: 10.1016/j.polymer.2006.08.059

Kang, Y.O., S.H. Choi, A. Gopalan, K.P. Lee, H.D. Kang and Y.S. Song, 2006. Tuning of morphology of Ag nanoparticles in the $\mathrm{Ag} /$ polyaniline nanocomposites prepared by Y-ray irradiation. J. Non-Crystal. Solids, 352: 463-468. DOI: 10.1016/j.jnoncrysol.2006.01.043

Karim, M.R., K.T. Lim, C.J. Lee, M.T. Islam Bhuiyan and H.J. Kim et al., 2007. Synthesis of core-shell silver-polyaniline nanocomposites by gamma radiolysis method. J. Polymer Sci. Part A: Polymer Chem., 45: 5741-5747. DOI: 10.1002/pola.22323

Khanna, P.K., N. Singh, S. Charan and A.K. Viswanath, 2005. Synthesis of $\mathrm{Ag} /$ polyaniline nanocomposite via an in situ photo-redox mechanism. Mate. Chemis Phys., 92: 214-219. DOI: 10.1016/j.matchemphys.2005.01.011

Lebaron, P.C., Z. Wang and T.J. Pinnavaia, 1999. Polymer-layered silicate nanocomposites: An overview. Applied Clay Sci., 15: 11-29. DOI: 10.1016/S0169-1317(99)00017-4

Liu, Z.H., X.J. Yang, Y. Majita and K. Ooi, 2006. Preparation of a polycation-intercalated layered manganese oxide nanocomposite by a delamination/reassembling process. Chem. Mater., 14: 4800-4806. http://pubs.acs.org/doi/abs/10.1021/cm020652h

Mbhele, Z.H., M.G. Salemane, C.G.C.E. Van Sittert, J.M. Nedeljkovic and V. Djokovic et al., 2003. Fabrication and characterization of silver-polyvinyl alcohol nanocomposites. Chem. Mater., 15: 5019-5024. DOI: $10.1021 / \mathrm{cm} 034505 \mathrm{a}$

Murray, C.B., D.J. Norris and M.G. Bawendi, 1993. Synthesis and characterization of nearly monodisperse $\mathrm{CdE}(\mathrm{E}=$ sulfur, selenium, tellurium) semiconductor nanocrystallites. J. Am. Chem. Soc., 115: 8706-8715. DOI: 10.1021/ja00072a 025

Niu, L., Q.H. Li, F.H. Wei, X. Wei and H. Wang, 2003. Electroactivity of electrochemically synthesized poly (aniline boronic acid) as a function of $\mathrm{pH}$ : Role of self-doping. J. Elect. Chem., 544: 121-125. DOI: $10.1021 / \mathrm{cm} 035296 \mathrm{x}$ 
Park, S.J., D.I. Seo and J.R. Lee, 2002. Surface modification of montmorillonite on surface acidbase characteristics of clay and thermal stability of epoxy/clay nanocomposites. J. Colloid Interface Sci., 251: 160-165. DOI: 10.1006/jcis.2002.8379

Porel, S., S. Singh, S.S. Harsha and D.N. Rao, 2005. Nanoparticle-embedded polymer: In situ synthesis, free-standing films with highly monodisperse silver nanoparticles and optical limiting. Chem. Mater., 17: 9-12. DOI: $10.1021 / \mathrm{cm} 0485963$

Qiao, Z., Y. Xie, X. Li, C. Wang, Y. Zhu and Y. Qian, 1999. $\gamma$-Irradiation preparation and phase control of nanocrystalline CdS. J. Mater. Chem., 9: 735-738. DOI: $10.1039 / \mathrm{a} 807757 \mathrm{f}$

Sarma, T.K., D. Chowdhury, A. Paul and A. Chattopadhyay, 2002. Synthesis of $\mathrm{Au}$ nanoparticle-conductive polyaniline composite using $\mathrm{H}_{2} \mathrm{O}_{2}$ a oxidizing as well as reducing agent. Chem. Commun., 14: 1048-1049. DOI: $10.1039 / b 201014 c$

Singh, R., K.S. Samra, R. Kumar and L. Singh, 2008. Microstructural modifications in swift ion irradiated PET. Radiat. Phys. Chem., 77: 575-580. DOI: 10.1016/j.radphyschem.2007.06.014

Steckenreitera, T., E. Balanzat, H. Fuess and C. Trautmann, 1997. Chemical modifications of PET induced by swift heavy ions. Nuclear Instruments Methods Phys. Res. Sect. B: Beam Interac. Mater. Atoms, 131: $\quad 159-166 . \quad$ DOI: $10.1016 / \mathrm{S} 0168-$ 583X(97)00364-9

Tjong, S.C. and G.D. Liang, 2005. Electrical properties of low-density polyethylene/ $\mathrm{ZnO}$ nanocomposites. Mater. Chem. Phys., 100: 1-5. DOI: 10.1016/j.matchemphys.2005.11.029
Virk, H.S. and A.K. Srivastava, 2001. Modification of optical, chemical and structural response of CR-39 polymer by $50 \mathrm{MeV}$ lithium ion irradiation. Radiat. Measur., 34: 65-67. DOI: 10.1016/S13504487(01)00122-6

Wu, W.T., Y. Wang, L. Shi, Q. Zhu and W. Pang et al., 2005. Fabrication of silver/cross-linked poly (vinyl alcohol) cable-like nanostructures under $\gamma$-ray irradiation. Nanotechnology, 16: 3017-3022. DOI: 10.1088/0957-4484/16/12/048

Yakuphanoglu, F., E. Basaran, B.F. Senkal and E. Sezer, 2006. Electrical and optical properties of an organic semiconductor based on polyaniline prepared by emulsion polymerization and fabrication of Ag/polyaniline/n-Si Schottky diode. J. Phys. Chem., 110: 16908-16913. DOI: 10.1021/jp060445v

VanZeghbroeck, B., 2007. Principles of semiconductor devices. Department of Electrical, Computer and Energy Engineering. http://www.chtm.unm.edu/sigmon/EE576/EE576.html

Zhengping, Q., Y. Xie, J. Huang, Y. Zhu and Y. Qian, 2000. Single-step confined growth of CdSe/polyacrylamide nanocomposites under girradiation. Radiat. Phys. Chem., 58: 287-292. DOI: 10.1016/S0969-806X(99)00465-X 